\title{
Beobachtung von Klima und Klimawandel in Mitteleuropa und Deutschland
}

Frank Kaspar, Hermann Mächel

3.1 Einleitung - 18

3.2 Beobachtung des Klimawandels in Deutschland - 18

3.2.1 Geschichte der Wetterbeobachtung in Deutschland - 18

3.2.2 Das aktuelle Stationsmessnetz in Deutschland - 19

3.2.3 Die Beobachtung wichtiger Klimagrößen im Einzelnen - 19

3.2.4 Klimatrends in Deutschland und den Bundesländern - 21

3.3 Datensätze für Deutschland und Europa - 21

3.3.1 Stationsdaten - 21

3.3.2 Gerasterte Datensätze - 23

3.4 Kurz gesagt - 24

Literatur - 24 


\subsection{Einleitung}

Der weltweite Klimawandel wirkt sich regional unterschiedlich aus. Um Klimaänderungen in Mitteleuropa und Deutschland genau zu beschreiben, benötigt man daher Beobachtungen und Klimamodelle mit größerer räumlicher Auflösung als bei globalen Betrachtungen ( Kap. 2). Für Deutschland und die Nachbarländer gibt es viele regionale Daten, sodass sich das hiesige Klima des vergangenen Jahrhunderts gut beschreiben lässt. Diese Datenbasis erlaubt daher auch eine Qualitätseinschätzung von Klimasimulationen auf der regionalen Skala ( Kap. 4). Zur Evaluation von regionalen Klimamodellen werden häufig atmosphärische, bodennahe Variable herangezogen, insbesondere Temperatur und Niederschlag, da von diesen direkte Auswirkungen auf die Gesellschaft ausgehen.

Die Entwicklung der Wetterbeobachtung ist eng mit der Geschichte der Wetterdienste verknüpft. Heute beobachtet der Deutsche Wetterdienst (DWD) das Wetter systematisch und international abgestimmt. Neben den Beobachtungsdaten der Wetterstationen werden zur Bewertung der Klimamodelle häufig aufbereitete Daten eingesetzt, die, ausgehend von den Beobachtungen, auf ein regelmäßiges räumliches Raster umgerechnet werden. Zusätzlich zu den traditionellen Beobachtungen wurde das Wetter während der vergangenen Jahrzehnte auch mit Satelliten und Wetterradaren beobachtet.

Die gesamten Daten erlauben Beschreibungen der Atmosphäre vom täglichen Wetter bis zu mehreren Jahrzehnten, über die sich das Klima ändert. Unter dem Begriff Klima versteht man dabei die statistische Beschreibung der relevanten Klimaelemente. Dabei muss ein ausreichend langer Zeitraum verwendet werden, sodass die statistischen Eigenschaften der Erdatmosphäre hinreichend genau charakterisiert werden. Gemäß den Empfehlungen der Weltmeteorologieorganisation (WMO 1959) werden daher bei der Berechnung von Klimagrößen üblicherweise drei aufeinanderfolgende Jahrzehnte verwendet. In der Vergangenheit wählte man überwiegend 1961 bis 1990, teilweise 1971 bis 2000 . Aufgrund der Klimaerwärmung ist die Zeit von 1961 bis 1990 aber nicht mehr repräsentativ für das aktuelle Klima (Scherrer et al. 2006). Für die Bewertung von Klimaänderungen ist aber weiterhin der ursprüngliche Referenzzeitraum (also 1961-1990) angemessen und wird durch die WMO für diesen Zweck nach wie vor empfohlen. Viele Anwendungen benötigen aber eine statistische Beschreibung des aktuellen Klimas. Für diesen Zweck wird daher die Verwendung eines aktuelleren Zeitraums empfohlen, und viele Wetterdienste stellen daher Auswertungen auch für den Vergleichszeitraum von 1981 bis 2010 zur Verfügung.

International entsteht im Rahmen des Global Climate Observing System (GCOS; Karl et al. 2010) ein langfristiges Beobachtungssystem. Dafür wurde eine Liste „essenzieller Klimavariablen“ definiert: Diese derzeit 50 Kenngrößen der Atmosphäre, des Ozeans und der Landoberfläche dienen einer ausführlichen Beschreibung des gesamten Klimasystems, sodass eine systematische langfristige Beobachtung dieser Kenngrößen angestrebt wird. Auch deutsche Institutionen leisten dazu umfangreiche Beiträge (Deutscher Wetterdienst 2013).

Seit einigen Jahrzehnten stehen auch Satellitendaten zur Verfügung. Aus ihnen lassen sich Datensätze verschiedener Klima- kenngrößen erstellen. Dabei ist aber zu berücksichtigen, dass die (frühen) Satelliteninstrumente nicht für diesen Zweck entwickelt wurden und daher zunächst die methodische Einheitlichkeit der Daten sichergestellt werden muss. Einige Projekte arbeiten an satellitenbasierten Datensätzen verschiedener essenzieller Klimavariable. So bearbeitet beispielsweise die Climate Change Initiative der Europäischen Weltraumorganisation ESA derzeit Datensätze von 13 Variablen (Hollmann et al. 2013). Die Europäische Organisation für die Nutzung meteorologischer Satelliten EUMETSAT erstellt im Rahmen ihrer Satellite Application Facility on Climate Monitoring Datensätze zu Strahlung, Wasserdampf und Bewölkung (siehe z. B. Karlsson et al. 2013).

\subsection{Beobachtung des Klimawandels in Deutschland}

\subsubsection{Geschichte der Wetterbeobachtung in Deutschland}

Seit jeher fasziniert das Wetter die Menschen und sie versuchten, ihre Beobachtungen in Bild und Wort festzuhalten - vor allem bei außergewöhnlichen Ereignissen. Doch erst mit der Erfindung von Messinstrumenten begann die objektive Wetteraufzeichnung (Schneider-Carius 1955, - Tab. 3.1). Im Jahr 1781 gründete sich in Mannheim die Pfälzische Meteorologische Gesellschaft Societas Meteorologica Palatina. Sie baute in Europa 39 Messstationen auf, 12 davon in Deutschland (Wege 2002) - alle mit den gleichen, geeichten Messinstrumenten, einer Anleitung sowie einheitlichen Formularen und Wettersymbolen. Die Messungen von Temperatur, Feuchte, Luftdruck, Sonnenschein und Niederschlag sowie die Schätzung von Bewölkung und Wind erfolgten dreimal täglich. Aus Geldmangel stellte diese Institution nach ein paar Jahren ihre Aktivitäten ein. Einige Beobachter führten die Messungen aber eigenständig weiter (Winkler 2006).

Es dauerte noch mehr als 50 Jahre, bis auf Initiative von Alexander von Humboldts 1848 der erste staatliche Wetterdienst in Preußen entstand (Hellmann 1887). Danach gründeten weitere Königreiche und Herzogtümer in Deutschland ihre eigenen Wetterdienste (Hellmann 1883). Allerdings wurden erst im Laufe der Zeit durch systematische Untersuchungen die Anforderungen an die Beobachtungen beschrieben und in den Beobachteranleitungen verbreitet. Weitere Verbesserungen gingen mit der Schulung der Laienbeobachter, einer repräsentativeren Auswahl der Beobachtungsstandorte und der technischen Entwicklung im Instrumentenbau einher.

Von Anfang an stützten sich die Wetterdienste auf Privatpersonen oder Institutionen, die schon vorher meteorologische Messungen durchführten. Diese Messungen waren aber sehr an deren individuelle Gegebenheiten angepasst, was sich in den unterschiedlichsten Beobachtungsterminen widerspiegelt. Beispielsweise konzentrierte sich das im Königreich Sachsen von der Forstwirtschaft errichtete Stationsnetz zur Untersuchung von Frostschäden vor allem auf die Minimumtemperatur und den Niederschlag, die beide mittags abgelesen wurden (Freydank 2013).

Nach dem Ersten Weltkrieg entstanden, bedingt durch den zunehmenden Flugverkehr, Flugwetterwarten mit Berufsbeob- 
Tab. 3.1 Wichtige Schritte auf dem Weg zur systematischen Klimabeobachtung

Erfindung des Barometers und Alkoholthermometers

$1643 / 1654$

Erste Klimaaufzeichnungen in Deutschland: individuelle, zum Teil unregelmäßige Beobachtungen

1700

Erstes europaweites, meteorologisches Messnetz von der Societas Meteorologica Palatina in Mannheim

$1781-1792$

Gründung staatlicher Wetterdienste

ab 1848

Gründung der Internationalen Meteorologieorganisation IMO, dadurch zunehmend Vereinheitlichung der Beobachtungen

1873

Deutsche Seewarte veröffentlicht täglich Bodenwetterkarten von Zentraleuropa und dem Atlantik, telegrafische Verbreitung von Wettermeldungen

Aufbau eines dichten Niederschlagsmessnetzes in Deutschland mit einheitlichen Messgeräten

1876

1880

Deutschlandweiter Wetterdienst, der die Beobachtungen weiter vereinheitlicht

1934

Gründung der Weltorganisation für Meteorologie (World Meteorological Organization, WMO)

1950

Automatisierung des Messnetzes des Deutschen Wetterdienstes achtern. Auch wurde es für die Wettervorhersagen immer wichtiger das Wetter gleichzeitig an vielen Standorten zu beobachten. Daher wurden Wetterwarten eingerichtet, die rund um die Uhr mit Berufsbeobachtern besetzt waren. Nebenbei schulten diese Profis die Laienbeobachter ihres Kreises. Viele Laienbeobachter hielten das tägliche Messen jedoch nicht lange durch, was häufig zu Stationsverlegungen und mehrmonatigen Lücken in den Messreihen führte. An fast allen Standorten gab es 1945 bei den Beobachtungen Unterbrechungen von Tagen bis mehreren Jahren (Mächel und Kapala 2013).

\subsubsection{Das aktuelle Stationsmessnetz in Deutschland}

Heute ist die Wetterbeobachtung durch einen gesetzlichen Auftrag geregelt: Der Deutsche Wetterdienst soll meteorologische Prozesse, Struktur und Zusammensetzung der Atmosphäre kurzfristig und langfristig erfassen, überwachen und bewerten. Dafür betreibt er ein Messnetz, archiviert die Beobachtungen, prüft deren Qualität und wertet sie aus (Deutscher Wetterdienst 2013). Zusammen mit den Beobachtungen der Vorgängerorganisationen ermöglichen diese Daten Aussagen darüber, wie sich das Klima in Deutschland entwickelt. Genug Daten für regionale Auswertungen liegen seit etwa 1881 vor (Kaspar et al. 2013). Die elektronischen Datenkollektive werden ständig ergänzt - auch durch die Digitalisierung historischer, täglicher Klimaaufzeichnungen aus Papierarchiven (Kaspar et al. 2015; Mächel et al. 2009; Brienen et al. 2013).

Kernstück des DWD-Messnetzes sind 182 hauptamtlich betriebene Wetterwarten und -stationen (Stand 01.11.2013). Der Geoinformationsdienst der Bundeswehr betreibt 31 weitere in das Netz integrierte Bodenwetterstationen. Darüber hinaus werden 1786 Mess- und Beobachtungsstationen ehrenamtlich betreut. Wetterradare gibt es an 19 Standorten, mit denen eine flächendeckende Niederschlagserfassung über Deutschland möglich ist. Messungen mit Radiosonden werden an 9 Stationen durchgeführt. Außerdem betreibt der DWD ein Netz mit 1267 phänologischen Beobachtungsstellen, an denen überwiegend ehrenamtliche Beobachter das Auftreten von Wachstumsphasen ausgewählter Pflanzenarten dokumentieren (Kaspar et al. 2014). Seit 2014 ist ein Großteil der Beobachtungsdaten frei zugänglich (www.dwd.de/cdc).

Zudem messen auch andere Institutionen und Privatpersonen verschiedene Klimavariable. Diese Daten fließen aber nur zu einem geringen Teil in die Datenbank des DWD oder in andere internationale Datensätze ein, weil sie oft nicht repräsentativ sind, die Anforderungen an das Messprogramm und die Dauerhaftigkeit des Betriebs nicht erfüllen oder datenpolitische Aspekte im Wege stehen.

Ein wichtiger Aspekt bei der Auswertung längerfristiger Trends ist die Homogenität der Messreihen. Veränderungen in den Messbedingungen können Messreihen inhomogen werden lassen. Es treten dann Sprünge auf, die nicht durch tatsächliche Klimaveränderungen verursacht wurden. Abhängig vom Messprinzip können die Ursachen der Inhomogenitäten sehr unterschiedlich sein. Müller-Westermeier (2004) hat die Homogenität deutscher Temperatur- und Niederschlagsreihen untersucht. Augter (2013) hat Vergleichsmessungen von automatischen und manuellen Messungen der Klimareferenzstationen verwendet, um die Auswirkungen der Automatisierung des Messnetzes zu analysieren. Der folgende Abschnitt diskutiert Einzelheiten der wichtigsten Parameter.

\subsubsection{Die Beobachtung wichtiger Klimagrößen im Einzelnen}

\section{- Temperatur}

Seit 60 Jahren wird an mehr als 500 Stationen die Temperatur gemessen. Zuvor war das Netz weniger dicht (Kaspar et al. 2015). Für die Zeit bis zum Zweiten Weltkrieg gibt es teilweise nur Monatswerte, viele Tageswerte gingen verloren. Weiter zurück bis 1881 liegen Monatswerte von mehr als 130 Stationen digitalisiert vor. Noch ältere Messreihen gibt es nur wenige, die aufgrund verschiedener Messverfahren und Beobachtungsprogramme meist inhomogen sind. Die längste dieser Reihen aus Berlin reicht bis 1719 zurück (Cubasch und Kadow 2011). Müller-Westermeier (2004) kommt bei der Untersuchung von Messreihen mit einer Dauer von mehr als 80 Jahren zu dem 
Ergebnis, dass die Mehrheit der Reihen eine oder mehrere Inhomogenität/en aufweist. Diese betrugen bis zu 1,7 K; wobei am häufigsten Inhomogenitäten von $0,2 \mathrm{~K}$ auftraten. In den meisten Fällen wurden dabei Stationsverlegungen als Ursache identifiziert. Ein weiterer wichtiger Faktor sind Veränderungen beim Strahlungsschutz der Messgeräte.

Zwischen 1995 und 2005 lösten elektrische Thermometer die visuell abzulesenden Quecksilberthermometer und Registriergeräte auf Bimetallbasis an den meisten Stationen ab. An ausgewählten Stationen wird weiter parallel analog gemessen (s. auch Augter 2013). Wesentliche Auswirkungen in den Zeitreihen von Monats- und Jahresmittelwerten haben sich dabei aber nicht ergeben. An allen Stationen mit Temperaturmessungen wird auch Feuchte gemessen.

\section{- Niederschlag}

Das DWD-Niederschlagsmessnetz besteht derzeit aus rund 1900 Messstellen. Seit etwa 60 Jahren liegen Tageswerte in hoher räumlicher Dichte vor, die in früheren Jahrzehnten teilweise aber noch deutlich höher war als heute. Von 1969 bis 2000 gab es mehr als 4000 Stationen. Monatswerte gibt es für die vergangenen 100 Jahre von mehr als 2000 Stationen, und zurück bis 1881 liegt noch ein Netz von mehreren 100 Stationen vor. Noch ältere Messreihen basieren auf sehr verschiedenen Messverfahren. Die längste durchgehende Niederschlagsreihe in Deutschland besitzt die Station Aachen, die seit 1844 in Betrieb ist. Müller-Westermeier (2004) fand bei der Untersuchung von 505 Niederschlagsmessreihen mit einer Dauer von mindestens 80 Jahren weniger Inhomogenitäten als im Fall der Temperatur, was aber auch durch die schwierigere Identifikation der Inhomogenitäten aufgrund der hohen Variabilität des Niederschlags bedingt ist. Die Inhomogenitäten lagen im Bereich von -30 bis $+40 \%$ und sind in den meisten Fällen (61\%) durch Stationsverlagerungen verursacht.

Seit etwa 1995 wird die Niederschlagsmessung zunehmend auf digitale Messsysteme umgestellt. Für diese Stationen liegen die Messungen zeitnah und in hoher zeitlicher Auflösung bis hin zu Minuten vor. Heute erfassen auch Wetterradare den Niederschlag. Durch Aneichung an Bodenniederschlagsstationen können flächendeckend, räumlich und zeitlich hoch aufgelöst Niederschlagsmengen abgeleitet werden.

\section{- Schneehöhe}

An den Niederschlagsstationen wird auch die Gesamtschneehöhe gemessen. In Bayern begannen diese Messungen bereits 1887, in den nördlichen Teilen Deutschlands erst gegen Ende der 1920er-Jahre. Ab etwa 1951 sind ausreichend digitale Schneehöhenangaben für ganz Deutschland vorhanden, obwohl diese an den Niederschlagsstationen in den alten Bundesländern erst ab 1979 vollständig digitalisiert vorliegen. Für die Zeit vor 1979 sind in den alten Bundesländern die Schneehöhen für die Klimastationen - d.h. Messstationen, an denen auch weitere Größen erfasst wurden - und einige nachträglich digitalisierte Niederschlagsstationen vorhanden. Mit der Automatisierung der Stationen ersetzten Schneehöhensensoren die manuellen Messungen.

\section{- Luftdruck}

Rund 210 Messstellen erfassen derzeit den Luftdruck. Vor 1950 gab es weniger Stationen und vor etwa 1930 nur einzelne Messreihen, die oft aufgrund verschiedener Messverfahren und Beobachtungsprogramme inhomogen sind. Die Messreihe des Observatoriums am Hohenpeißenberg begann 1781. Zwischen 1995 und 2005 ersetzten digitale Geräte weitgehend die Quecksilber- und Dosenbarometer - ohne wesentliche Inhomogenitäten in den Zeitreihen.

\section{- Wind}

Seit etwa 20 Jahren messen rund 300 Stationen den Wind. Dazu kommen Windschätzungen von den nebenamtlich betriebenen Stationen. Vor 1950 gab es nur einzelne Messreihen. Zeitreihen von Windschätzungen gehen teilweise bis ins 19. Jahrhundert zurück, sind aber wegen unterschiedlicher Mess- und Auswertemethoden nur bedingt für längerfristige Auswertungen nutzbar.

\section{- Sonnenscheindauer}

Wie lange die Sonne scheint, erfassen seit 60 Jahren rund 300 Stationen. Davor gab es nur einzelne, häufig inhomogene Messreihen. Ursprünglich wurden die Messungen auf der Basis des Brennglaseffekts durchgeführt, visuell ausgewertet und stündlich dokumentiert. Zwischen 1995 und 2005 wurde das Messnetz weitgehend auf automatische Messgeräte umgestellt, die mit hoher zeitlicher Auflösung arbeiten. Aufgrund des grundsätzlich anderen Messprinzips sind hier stärkere Inhomogenitäten durch die Automatisierung festgestellt worden als bei anderen Größen (Augter 2013).

\section{- Wolken}

An 64 Wetterstationen des DWD und 31 Stationen des Geoinformationsdienstes der Bundeswehr werden Wolkenart, Bedeckungsgrad und Wolkenuntergrenze visuell erfasst und dokumentiert. Die Zeitreihen reichen zurück bis in die 1940er-Jahre, an einigen Stationen sogar bis ins 19. oder 18. Jahrhundert. Seit den 1990er-Jahren dienen Laser-Ceilometer dazu, die Wolkenbedeckung und die Wolkenuntergrenze genau zu bestimmen.

Weiterhin gibt es inzwischen ausreichend lange Beobachtungen per Wettersatelliten, um daraus Datensätze etwa für Bedeckungsgrad, Wolkentyp, optische Dicke, Wolkenphase und Wolkenobergrenze abzuleiten, z. B. in der Satellite Application Facility on Climate Monitoring (Karlsson et al. 2013) oder der Climate Change Initiative der ESA.

\section{- Strahlung}

Strahlung wird an 121 Stationen gemessen. Dabei kommen allerdings Messinstrumente unterschiedlicher Qualität zum Einsatz. Als höherwertig werden Pyranometer angesehen (Becker und Behrens 2012). Diese messen an 28 Stationen die kurzwellige Globalstrahlung und langwellige Wärmestrahlung der Atmosphäre sowie zusätzlich die diffuse Sonneneinstrahlung. An neun dieser Stationen erfassen auch Pyrgeometer die Wärmestrahlung der Atmosphäre. Gespeichert werden Ein-Minuten-Mittelwerte. An neun Stationen liegen Messreihen der Globalstrahlung von mindestens 50 Jahren vor. Auch bei der bodennahen Strahlung lassen sich Satellitendaten nutzen (Posselt et al. 2012). 
Tab. 3.2 Temperatur- und Niederschlagstrends in Deutschland in verschiedenen Zeiträumen

\begin{tabular}{|l|l|l|l|l|l|}
\hline Intervall & Frühling & Sommer & Herbst & Winter & Jahr
\end{tabular}

Temperaturtrend in ${ }^{\circ} \mathrm{C}$ pro Dekade (links) sowie über den angegebenen Zeitraum (rechts)

\begin{tabular}{|l|l|l|l|l|l|l|l|l|l|}
\hline $1881-2014$ & 0,11 & 1,4 & 0,09 & 1,2 & 0,10 & 1,3 & 0,09 & 1,1 & 0,10 \\
\hline $1901-2014$ & 0,10 & 1,1 & 0,11 & 1,2 & 0,11 & 1,3 & 0,07 & 0,8 & 0,10 \\
\hline $1951-2014$ & 0,30 & 1,9 & 0,25 & 1,6 & 0,15 & 0,9 & 0,28 & 1,8 & 0,24 \\
\hline
\end{tabular}

Niederschlagstrend in mm pro Dekade (links in jeder Spalte) sowie in \% pro Dekade relativ zum Mittelwert 1961-1990 (rechts in jeder Spalte)

\begin{tabular}{|c|c|c|c|c|c|c|c|c|c|}
\hline $1881-2014$ & 1,5 & 0,8 & $-0,1$ & 0,0 & 1,0 & 0,6 & 3,6 & 2,0 & 6,1 \\
\hline $1901-2014$ & 1,6 & 0,8 & 0,1 & 0,0 & 1,5 & 0,8 & 3,0 & 1,7 & 6,2 \\
\hline $1951-2014$ & 1,4 & 0,8 & $-2,2$ & $-0,9$ & 3,5 & 1,9 & 4,1 & 2,3 & 6,5 \\
\hline
\end{tabular}

Mittelwert des Niederschlags für die Periode 1961-1990 in mm

\subsubsection{Klimatrends in Deutschland und den Bundesländern}

Aus den Beobachtungen der Messstationen lässt sich ableiten, wie sich das Klima in Deutschland in den vergangenen 130 Jahren verändert hat, auch speziell in einzelnen Regionen. Regelmäßig aktualisiert der Deutsche Wetterdienst ausgehend von diesen Daten seine Auswertungen, beispielsweise in Form von Karten im Deutschen Klimaatlas. Aus den Karten lassen sich Mittelwerte und Trends für Gesamtdeutschland, die Bundesländer oder andere Regionen berechnen (Kaspar et al. 2013). Dabei wird wie folgt vorgegangen: Zunächst werden die beobachteten Werte zeitlich gemittelt. Dann wird ein Rasterfeld mit einer Auflösung von $1 \mathrm{~km}^{2}$ erzeugt - dabei wird die Höhenabhängigkeit der Klimagrößen berücksichtigt (Müller-Westermeier 1995; Maier et al. 2003). Dieses Rasterfeld dient dann dazu, Mittelwerte für bestimmte Regionen zu berechnen. Im Vergleich zu einer reinen Mittelwertbildung aus den Stationsdaten reduziert diese Vorgehensweise die Auswirkungen, die Veränderungen im Messnetz auf die Ergebnisse haben. Auch der Effekt von Inhomogenitäten einzelner Stationsreihen, z. B. infolge von Verlegung, wird reduziert. Daten für dieses Verfahren liegen für Temperatur und Niederschlag für die Zeit seit 1881 und für Sonnenscheindauer seit 1951 ausreichend vor. - Abb. 3.1 zeigt Ergebnisse dieser Auswertungen.

Von 1881 bis 2014 stieg die Temperatur deutlich, sowohl im Jahresdurchschnitt $\left(+1,3^{\circ} \mathrm{C}\right)$ als auch im Sommer $\left(+1,2^{\circ} \mathrm{C}\right)$ und Winter $\left(+1,1^{\circ} \mathrm{C}\right)$. Damit erwärmte sich Deutschland mehr als die Erde im Durchschnitt. Im Westen Deutschlands stieg die Temperatur etwas stärker als im Osten.

Die Resultate stimmen mit früheren Auswertungen anderer Autoren überein: Auch der Klima-Trendatlas Deutschland 1901 bis 2000 zeigt Trends der mittleren Temperatur in Deutschland. Allerdings wurden Daten von weniger Stationen verwendet (Schönwiese und Janoschitz 2008; Schönwiese et al. 2004). Für den Zeitraum 1901 bis 2000 erhalten Schönwiese et al. (2004) für Deutschland einen linearen Trend von $+1^{\circ} \mathrm{C}$.

Da die Temperatur stets schwankt, bestimmt der Startzeitpunkt der Berechnung die Stärke des Trends. Ebenso fällt der Trend je nach Länge des Zeitraums mehr oder weniger stark aus. Er ist stärker, wenn man Zeiträume in der zweiten Hälfte des 20. Jahrhunderts wählt ( $\mathbf{\bullet}$ Tab. 3.2). Die wärmsten Jahre von 1881 bis 2014 in Deutschland waren 2000, 2007 und 2014, die kältesten 1940, 1956 und 1888.

Die Niederschläge haben von 1881 bis 2014 um 10,2\% zugenommen, verglichen mit dem langjährigen Mittel von 1961 bis 1990. Im Winter stieg die Niederschlagsmenge um $26 \%-$ dabei mehr im Westen Deutschlands als im Osten. Im Sommer gab es dagegen 0,6\% weniger Niederschläge. Seit 1951 nahm die jährliche Sonnenscheindauer deutschlandweit um etwa $4 \% \mathrm{zu}$. Um einen Vergleich über unterschiedliche Zeiträume zu ermöglichen, sind die prozentualen Änderungen in - Tab. 3.2 jeweils pro Jahrzehnt angegeben.

Wie signifikant ein Trend ausfällt, hängt von der betrachteten Klimagröße, der Region, der Jahreszeit und dem Zeitraum der Auswertung ab. Dies wurde mit vielen Details von Rapp (2000) ausgewertet: In den 100 Jahren von 1896 bis 1995 erwärmte sich Deutschland überwiegend statistisch signifikant. Niederschläge nahmen besonders im Winterhalbjahr signifikant zu und daraus resultierend auch ganzjährig, vor allem im Westen des Landes. Vergleichbar signifikant sind die Trends der deutschlandweiten Mittelwerte in $\mathbf{D}$ Abb. 3.1.

\subsection{Datensätze für Deutschland und Europa}

\subsubsection{Stationsdaten}

Der niederländische Wetterdienst KNMI sammelt und aktualisiert Daten europäischer Wetterstationen im Projekt European Climate Assessment and Data (ECA $\sim D)$ (Klok und Klein-Tank 2008). Der Datenbestand dieses Projekts basiert auf Zulieferungen von Wetterdiensten, Observatorien und Universitäten aus 62 Ländern in Europa, im Mittelmeerraum und Vorderasien. Dabei handelt es sich um tägliche Daten von zwölf meteorologischen Kenngrößen: Minimum-, Mittel- und Maximaltemperatur, Niederschlagsmenge, Sonnenscheindauer, Wolkenbedeckung, Schneehöhe, Luftfeuchtigkeit, Windgeschwindigkeit, Windspitze, Windrichtung und Luftdruck. Derzeit liegen 37.025 


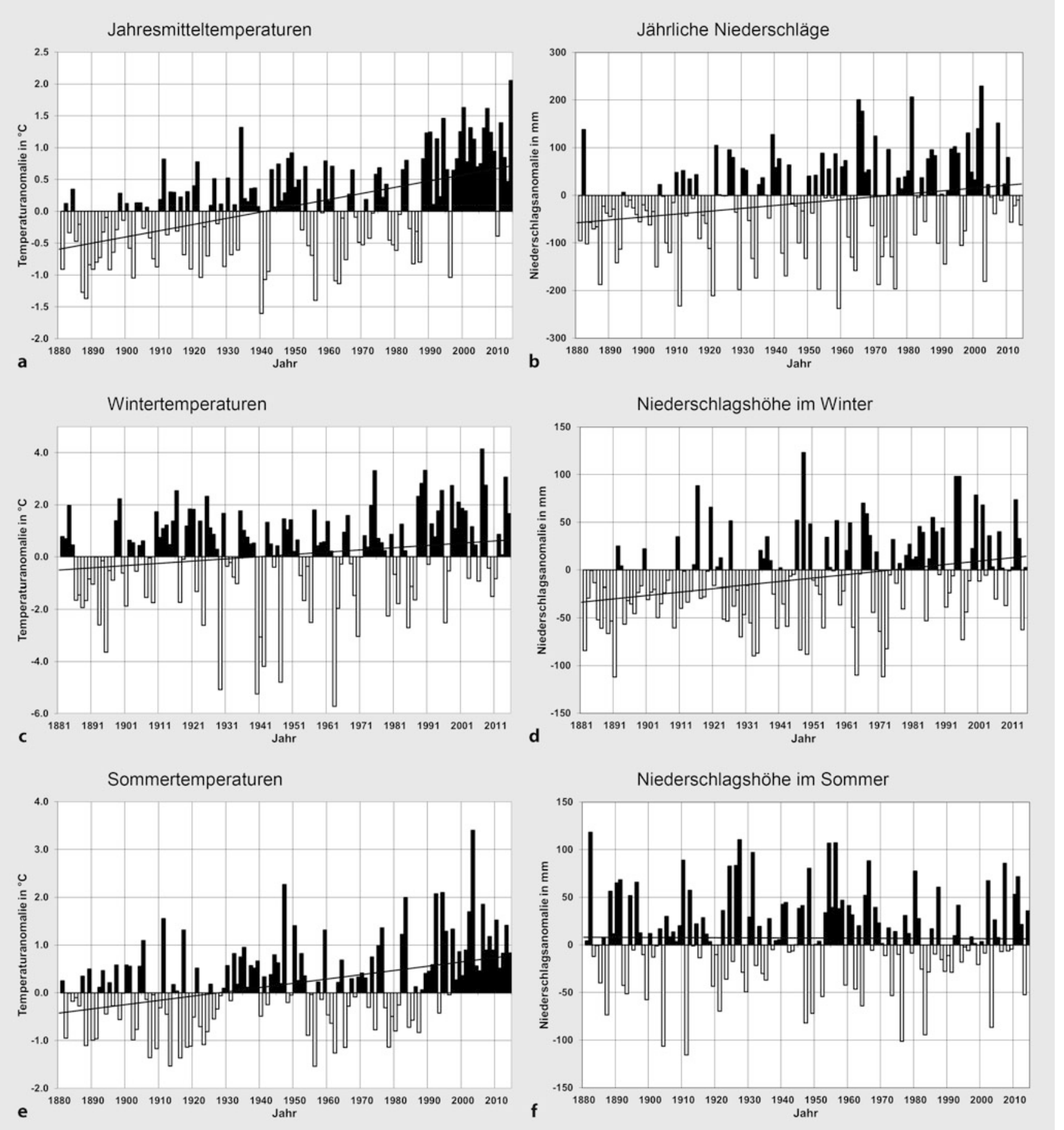

- Abb. 3.1 Trends der Temperatur und Niederschlagshöhe in Deutschland von 1881 bis 2014 jeweils als Abweichung vom Mittelwert des Zeitraums 1961 bis 1990. a Jahresmitteltemperaturen: Der lineare Trend von insgesamt $1,3^{\circ} \mathrm{C}$ innerhalb von 134 Jahren ist statistisch hoch signifikant ( $p$-Wert $\left.<0,001\right)$. b Jährliche Niederschläge: Der lineare Trend über die Gesamtzeit ist statistisch hoch signifikant (Zunahme um 81 mm; p-Wert 0,004). c Wintertemperaturen: Der lineare Trend von insgesamt $1,1^{\circ} \mathrm{C}$ innerhalb von 134 Jahren ist statistisch gering signifikant ( $p$-Wert 0,03). $\mathbf{d}$ Niederschlagshöhe im Winter: Der lineare Trend über die Gesamtzeit ist statistisch hoch signifikant (Zunahme um $47 \mathrm{~mm}$; p-Wert $<0,001$ ). e Sommertemperaturen in Deutschland: Der lineare Trend von insgesamt $1,2^{\circ} \mathrm{C}$ innerhalb von 134 Jahren ist statistisch hoch signifikant ( $p$-Wert $<0,001$ ). f Niederschlagshöhe im Sommer: Es besteht kein statistisch signifikanter linearer Trend über die Gesamtzeit (Abnahme um 1,5 mm; p-Wert 0,9). Sommer bezieht sich jeweils auf Juni bis August. Winter bezieht sich auf Dezember bis Februar und den Zeitraum von 1881/82 bis 2014/15 
- Tab. 3.3 Klimatologische Rasterdaten für Deutschland und angrenzende Gebiete

\begin{tabular}{|c|c|c|c|c|c|}
\hline $\begin{array}{l}\text { Datensatz/ } \\
\text { Projekt }\end{array}$ & Parameter & Gebiet & Räumliche Auflösung & $\begin{array}{l}\text { Zeitraum und zeitliche } \\
\text { Auflösung }\end{array}$ & Referenz \\
\hline $\mathrm{E}-\mathrm{OBS}$ & $\begin{array}{l}\text { Temperatur, Nie- } \\
\text { derschlag, Druck }\end{array}$ & Europa & $25 \mathrm{~km}$ & Ab 1950; täglich & $\begin{array}{l}\text { Haylock et al. (2008), } \\
\text { van den Besselaar } \\
\text { et al. (2011) }\end{array}$ \\
\hline REGNIE & Niederschlag & Deutschland & Ca. 1 km & $\begin{array}{l}\text { Ab 1931: alte Bundes- } \\
\text { länder; ab 1951: alle } \\
\text { Bundesländer; täglich }\end{array}$ & Rauthe et al. (2013) \\
\hline HYRAS & Niederschlag & $\begin{array}{l}\text { Deutsche Fluss- } \\
\text { einzugsgebiete mit } \\
\text { Nachbarländer }\end{array}$ & $1 \mathrm{~km}$ & 1951-2006, täglich & Rauthe et al. (2013) \\
\hline $\begin{array}{l}\text { DWD-Klimaü- } \\
\text { berwachung }\end{array}$ & $\begin{array}{l}\text { Niederschlag, Tem- } \\
\text { peratur, Sonnen- } \\
\text { scheindauer }\end{array}$ & Deutschland & $1 \mathrm{~km}$ & $\begin{array}{l}1881 \text { bis heute, } \\
\text { Sonnenscheindauer ab } \\
\text { 1951; monatlich }\end{array}$ & Kaspar et al. (2013) \\
\hline STAMMEX & Niederschlag & Deutschland & $\begin{array}{l}1931 \text { bis heute in } 0,5^{\circ} ; \\
1951 \text { bis heute in } 0,5^{\circ} ; 0,25^{\circ} ; \\
1971-2000 \text { in } 0,5^{\circ} ; 0,25^{\circ} ; 0,1^{\circ}\end{array}$ & Jeweils täglich & Zolina et al. (2014) \\
\hline DEKLIM & Wind & Deutschland & $1 \mathrm{~km}$ & 1951-2001, monatlich & Walter et al. (2006) \\
\hline HISTALP & $\begin{array}{l}\text { Temperatur, Nie- } \\
\text { derschlag }\end{array}$ & Alpen & $5 \mathrm{~min}$ & $\begin{array}{l}\text { Temperatur (1780- } \\
\text { 2009), Niederschlag } \\
\text { (1801-2003), monatlich }\end{array}$ & $\begin{array}{l}\text { Chimani et al. } \\
(2013,2011)\end{array}$ \\
\hline ALPIMP & Niederschlag & Alpen & $1 / 6$ Grad & 1800-2003, monatlich & $\begin{array}{l}\text { Efthymiadis et al. } \\
\text { (2006) }\end{array}$ \\
\hline EURO4M/Alpine & Niederschlag & Alpen & $5 \mathrm{~km}$ & 1971-2008, täglich & Isotta et al. (2013) \\
\hline
\end{tabular}

Zeitreihen von 7848 meteorologischen Stationen vor. Allerdings variiert die Menge der bereitgestellten Daten aus den einzelnen Ländern erheblich. Für Deutschland liegt mit 1084 Stationen eine vergleichsweise hohe Datendichte vor (Stand aller Angaben 26.01.2014, abgerufen unter ecad.knmi.nl). Für wissenschaftliche Zwecke sind $61 \%$ der Daten frei zugänglich. Die Daten des ECA\&D-Bestands wurden nicht homogenisiert, da für tägliche Daten derzeit keine automatischen Verfahren verfügbar sind. Da bei Trendanalysen, insbesondere im Fall von Extremwerten, die Homogenität der Zeitreihe beachtet werden muss, wurden zumindest Tests der Homogenität durchgeführt: In einer Untersuchung von Wijngaard et al. (2003) wurde für den Zeitraum von 1901 bis 1999 die Homogenität von 94\% der Temperaturreihen und $25 \%$ der Niederschlagsreihen als ,suspekt“" oder „zweifelhaft“ bewertet. Weitere Untersuchungen zur Homogenität führten ebenfalls zu dem Ergebnis, dass sich die Anzahl der als homogen eingestuften Reihen stark zwischen den Klimagrößen unterscheidet: Für den Zeitraum von 1960 bis 2004 wurden dabei zwischen $12 \%$ (Minimumtemperatur) und $59 \%$ (Niederschlag) der Reihen als homogen bewertet (Begert et al. 2008).

\subsubsection{Gerasterte Datensätze}

Die oben beschriebenen Routineanalysen dienen vor allem dazu, langfristige Trends zu bestimmen und eine Einordnung der aktuellen Monate vorzunehmen. Darüber hinaus gibt es für andere Zwecke weitere gerasterte Datensätze. Diese basieren für die Region Deutschland üblicherweise ebenfalls auf den Beobachtungen des DWD, können sich aber, etwa in Bezug auf die ausgewählten Messstationen, zusätzlich genutzte Datenquellen oder die Methodik unterscheiden. Datensätze, die mehr als Deutschland abdecken, enthalten vergleichbare Informationen aus den Messnetzen der Nachbarländer. Aufgrund datenpolitischer Einschränkungen basieren diese aber oft auf einer deutlich geringeren Stationsdichte als vergleichbare nationale Datensätze.

Ein häufig genutzter gerasterter Datensatz für Europa ist der Datensatz E-OBS, der auf den Stationsdaten des ECA\&D-Projekts basiert. Als Rasterprodukte stehen Temperatur und Niederschlag (Haylock et al. 2008) sowie Luftdruck (van den Besselaar et al. 2011) zur Verfügung. Für die Bewertung regionaler Klimamodelle und der Analyse von Extremereignissen ist er allerdings nur eingeschränkt verwendbar (Hofstra et al. 2009). Bei geringer Stationsdichte sind die Rasterdaten stark geglättet (Hofstra et al. 2010), was insbesondere bei der Bewertung von Trends in Extremen berücksichtigt werden muss. Im Vergleich zu einem höher aufgelösten nationalen Datensatz für Großbritannien zeigen Maraun et al. (2012), dass sich vor allem extreme Niederschläge in bergigen und datenarmen Regionen mit dem E-OBS-Datensatz nicht gut untersuchen lassen. Kyselý und Plavcová (2010) zeigen, dass sich Minimum- und Maximumtemperatur des EOBS-Datensatzes und eines nationalen Rasterdatensatzes für die Tschechische Republik aufgrund der unterschiedlichen Stationsdichte deutlich unterscheiden.

- Tab. 3.3 gibt eine Übersicht über neuere Datensätze, die für Überprüfung von Modellen in Deutschland und angrenzenden Regionen relevant sind. 
- Tab. 3.4 Ausgewählte Informationsportale

Klimaatlas des Deutschen Wetterdienstes

Klimaatlas der regionalen Klimabüros der Helmholtz-Gemeinschaft

Norddeutscher Klimaatlas des Norddeutschen Klimabüros

Klimaatlas Nordrhein-Westfalen

Klimaatlas Baden-Württemberg

Norddeutscher Klimamonitor

Umweltatlas Hessen

Umweltatlas Berlin www.deutscher-klimaatlas.de

- www.regionaler-klimaatlas.de

- www.norddeutscher-klimaatlas.de

- www.klimaatlas.nrw.de

- www4.lubw.baden-wuerttemberg.de/servlet/is/16703

- www.norddeutscher-klimamonitor.de

- atlas.umwelt.hessen.de/atlas/

- www.stadtentwicklung.berlin.de/umwelt/umweltatlas
Der Datensatz HYRAS deckt die deutschen Flusseinzugsgebiete inklusive der zugehörigen Regionen der Nachbarländer ab. Er basiert auf insgesamt 6200 Stationen (Rauthe et al. 2013). Durch seine räumliche Auflösung von $1 \mathrm{~km}$ verfügt er über eine deutlich andere Häufigkeitsverteilung für Niederschläge als der E-OBS-Datensatz mit einer Auflösung von $25 \mathrm{~km}$. Dabei stimmt die Verteilung im HYRAS-Datensatz gut mit der Verteilung überein, die direkt aus Stationen abgeleitet wird.

Im STAMMEX-Projekt wurde bei der Erzeugung der Datensätze versucht, eine gleichbleibende Stationsdichte zu erreichen. Es wurden tägliche Raster in unterschiedlicher räumlicher Auflösung erzeugt.

Für die Alpen realistische Rasterfelder des Niederschlags zu erzeugen ist aufgrund ihrer komplexen Oberflächenstruktur besonders schwierig. Daher wurde diese Fragestellung in mehreren Projekten behandelt (HISTALP, ALPIMP, EURO4M).

Klimatologische Informationen enthalten auch Klimaatlanten. Diese gibt es sowohl für Deutschland (z. B. Deutscher Wetterdienst 1999, 2001, 2003, 2006) als auch für einzelne Regionen (z. B. Oberrheinische Universitäten 1996). Im Internet finden sich zudem verschiedene interaktive Klimaatlanten, die teilweise auch Ergebnisse aus Szenarienrechnungen enthalten ( $\mathbf{\bullet}$ Tab. 3.4).

\subsection{Kurz gesagt}

Erste systematische Wetterbeobachtungen gab es bereits im 18. Jahrhundert. Aber nur an einzelnen Standorten wurden sie kontinuierlich fortgesetzt. Mit der Gründung staatlicher Wetterdienste im 19. Jahrhundert begannen umfangreichere Beobachtungen. Heute beobachten die Wetterdienste in Deutschland und den Nachbarländern, wie sich das Klima in Mitteleuropa verändert. Auf Basis der gesammelten Beobachtungen lassen sich Aussagen über die Klimaentwicklung in Deutschland treffen: Von 1881 bis 2014 stiegen die mittleren Temperaturen in Deutschland deutlich, sowohl im Jahresdurchschnitt $\left(+1,3^{\circ} \mathrm{C}\right)$ als auch im Sommer $\left(+1,2^{\circ} \mathrm{C}\right)$ und Winter $\left(+1,1^{\circ} \mathrm{C}\right)$. In diesem Zeitraum haben die jährlichen Niederschläge um 10,2\% zugenommen (im Vergleich zum langjährigen Mittelwert 1961-1990). Die Zunahme wird überwiegend durch die Zunahme der Winterniederschläge um $26 \%$ verursacht. Aus den Beobachtungen lassen sich auch Datensätze ableiten, mit denen sich regionale Klima- modelle überprüfen lassen. Dabei sind allerdings die spezifischen Eigenschaften der Datensätze zu berücksichtigen, die sich etwa aus der unterschiedlichen Stationsdichte ergeben. Insbesondere bei der Betrachtung von Extremen und Trends sind regionale Datensätze mit hoher Stationsdichte vorteilhaft.

\section{Literatur}

Augter G (2013) Vergleich der Referenzmessungen des Deutschen Wetterdienstes mit automatisch gewonnenen Messwerten, 2. Aufl. Berichte des Deutschen Wetterdienstes, Bd. 238.

Becker R, Behrens K (2012) Quality assessment of heterogeneous surface radiation network data. Adv Sci Res 8:93-97. doi:10.5194/asr-8-93-2012

Begert M, Zenkusen E, Haeberli C, Appenzeller C, Klok L (2008) An automated homogenization procedure; performance assessment and application to a large European climate dataset. Meteor Z 17(5):663-672

Besselaar EJM van den, Haylock MR, Schrier G van der, Klein Tank AMG (2011) An European daily high-resolution observational gridded data set of sea level pressure. J Geophys Res 116:D11110. doi:10.1029/2010JD015468

Brienen S, Kapala A, Mächel H, Simmer C (2013) Regional centennial precipitation variability over Germany from extended observation records. Int J Climatol 33. doi:10.1002/joc.3581

Chimani B, Böhm R, Matulla C, Ganekind M (2011) Development of a longterm dataset of solid/liquid precipitation. Adv Sci Res 6:39-43

Chimani B, Matulla C, Böhm R, Hofstätter M (2013) A new high resolution absolute temperature grid for the Greater Alpine Region back to 1780. Int J Climatol 33:2129-2141. doi:10.1002/joc.3574

Cubasch U, Kadow C (2011) Global climate change and aspects of regional climate change in the Berlin-Brandenburg region. Die Erde 142:3-20

Deutscher Wetterdienst (1999) Klimaatlas Bundesrepublik Deutschland. Teil 1 Lufttemperatur, Niederschlagshöhe, Sonnenscheindauer. Selbstverlag des Deutschen Wetterdienstes, Offenbach

Deutscher Wetterdienst (2001) Klimaatlas Bundesrepublik Deutschland. Teil 2 Verdunstung, mittlere tägliche Extremwerte, Kontinentalität. Selbstverlag des Deutschen Wetterdienstes, Offenbach

Deutscher Wetterdienst (2003) Klimaatlas Bundesrepublik Deutschland. Teil 3 Bewölkung, Globalstrahlung, Tage mit Überschreitung klimatologischer Schwellenwerte, Phänologie. Selbstverlag des Deutschen Wetterdienstes, Offenbach

Deutscher Wetterdienst (2006) Klimaatlas Bundesrepublik Deutschland. Teil 4 Klimatische Wasserbilanz, Tägliche Temperaturschwankung, Windgeschwindigkeit, Dampfdruck, Schneedecke. Selbstverlag des Deutschen Wetterdienstes, Offenbach

Deutscher Wetterdienst (2013) Die deutschen Klimabeobachtungssysteme. Inventarbericht zum Global Climate Observing System (GCOS). Selbstverlag des Deutschen Wetterdienstes, Offenbach (http://www.gcos.de/ inventarbericht) 
Efthymiadis D, Jones PD, Briffa KR, Auer I, Böhm R, Schöner W, Frei C, Schmidli $J$ (2006) Construction of a 10-min-gridded precipitation data set for the Greater Alpine Region for 1800-2003. J Geophys Res 111:D01105. doi:10.1029/2005JD006120

Freydank E (2013) 150 Jahre staatliches meteorologisches Messnetz in Sachsen. In: Goethes weiteres Erbe: 200 Jahre Klimastation Jena. Beiträge des Jubiläumskolloquiums „200 Jahre Klimamessstation Jena“. Annalen d Meteorol, Bd. 46. Selbstverlag des Deutschen Wetterdienstes, Offenbach a M, S 172

Haylock MR, Hofstra N, Klein Tank AMG, Klok EJ, Jones PD, New M (2008) A European daily high-resolution gridded dataset of surface temperature and precipitation for 1950-2006. J Geophys Res (Atmospheres) 113:D20119. doi:10.1029/2008JD10201

Hellmann G (1883) Repertorium der deutschen Meteorologie: Leistungen der Deutschen in Schriften, Erfindungen und Beobachtungen auf dem Gebiet der Meteorologie und dem Erdmagnetismus von den ältesten Zeiten bis zum Schluss des Jahres 1881. Engelmann-Verlag, Leipzig (XXIV: 996)

Hellmann G (1887) Geschichte des Königl. Preuß Meteorologischen Instituts von seiner Gründung im Jahre 1847 bis Reorganisation im Jahre 1885. In: Ergebnisse der Meteorologischen Beobachtungen im Jahre 1885. Königlich Preußisches Meteorologisches Institut, Berlin, S XX-LXIX

Hofstra N, Haylock M, New M, Jones PD (2009) Testing E-OBS European highresolution gridded data set of daily precipitation and surface temperature. J Geophys Res 114:D21101

Hofstra N, New M, McSweeney C (2010) The influence of interpolation and station network density on the distributions and trends of climate variables in gridded daily data. Climate Dynamics 35(5):841-858

Hollmann R, Merchant CJ, Saunders R, Downy C, Buchwitz M, Cazenave A, Chuvieco E, Defourny P, de Leeuw G, Forsberg R, Holzer-Popp T, Paul F, Sandven S, Sathyendranath S, van Roozendael M, Wagner W (2013) The ESA Climate Change Initiative: Satellite data records for essential climate variables. Bull Amer Meteor Soc 94:1541-1552. doi:10.1175/BAMS-D-11-00254.1

Isotta FA, Frei C, Weilguni V, Perčec Tadić M, Lassègues P, Rudolf B, Pavan V, Cacciamani C, Antolini G, Ratto SM, Munari M, Micheletti S, Bonati V, Lussana C, Ronchi C, Panettieri E, Marigo G, Vertačnik G (2013) The climate of daily precipitation in the Alps: development and analysis of a high-resolution grid dataset from pan-Alpine rain-gauge data. Int J Climatol. doi:10.1002/ joc.3794

Karl TR, Diamond HJ, Bojinski S, Butler JH, Dolman H, Haeberli W, Harrison DE, Nyong A, Rösner S, Seiz G, Trenberth K, Westermeyer W, Zillman J (2010) Observation needs for climate information, prediction and application: Capabilities of Existing and Future Observing Systems. Procedia Environmental Sciences 1:192-205. doi:10.1016/j.proenv.2010.09.013

Karlsson KG, Riihelä A, Müller R, Meirink JF, Sedlar J, Stengel M, Lockhoff M, Trentmann J, Kaspar F, Hollmann R, Wolters E (2013) CLARA-A1: a cloud, albedo, and radiation dataset from $28 \mathrm{yr}$ of global AVHRR data. Atmos Chem Phys 13:5351-5367. doi:10.5194/acp-13-5351-2013

Kaspar F, Müller-Westermeier G, Penda E, Mächel H, Zimmermann K, KaiserWeiss A, Deutschländer T (2013) Monitoring of climate change in Germany - data, products and services of Germany's National Climate Data Centre. Adv Sci Res 10:99-106. doi:10.5194/asr-10-99-2013

Kaspar F, Zimmermann K, Polte-Rudolf C (2014) An overview of the phenological observation network and the phenological database of Germany's national meteorological service (Deutscher Wetterdienst). Adv Sci Res 11:93-99. doi:10.5194/asr-11-93-2014

Kaspar F, Tinz B, Mächel H, Gates L (2015) Data rescue of national and international meteorological observations at Deutscher Wetterdienst. Adv Sci Res 12:57-61. doi:10.5194/asr-12-57-2015

Klok EJ, Klein Tank AMG (2008) Updated and extended European dataset of daily climate observations. Int J Climatol 29:1182. doi:10.1002/joc.1779

Kyselý J, Plavcová E (2010) A critical remark on the applicability of E-OBS. European gridded temperature data set for validating control climate simulations. doi:10.1029/2010JD014123

Mächel H, Kapala A (2013) Bedeutung langer historischer Klimareihen. In: Goethes weiteres Erbe: 200 Jahre Klimastation Jena. Beiträge des Jubiläumskolloquiums „200 Jahre Klimamessstation Jena“. Annalen d Meteorol, Bd. 46. Selbstverlag des Deutschen Wetterdienstes, Offenbach a M, S 172
Mächel H, Kapala A, Behrendt J, Simmer C (2009) Rettung historischer Klimadaten in Deutschland: das Projekt KLIDADIGI des DWD. Klimastatusbericht 2008. Deutscher Wetterdienst, Offenbach/Main

Maier U, Kudlinski J, Müller-Westermeier G (2003) Klimatologische Auswertung von Zeitreihen des Monatsmittels der Lufttemperatur und der monatlichen Niederschlagshöhe im 20. Jahrhundert. Berichte des Deutschen Wetterdienstes, Bd. 223. Selbstverlag des Deutschen Wetterdienstes, Offenbach am Main

Maraun D, Osborn TJ, Rust HW (2012) The influence of synoptic airflow on UK daily precipitation extremes. Part II: regional climate model and E-OBS data validation. Climate Dynamics 39(1-2):287-301

Müller-Westermeier G (1995) Numerisches Verfahren zur Erstellung klimatologischer Karten. Berichte des Deutschen Wetterdienstes, Bd. 193. Selbstverlag des Deutschen Wetterdienstes, Offenbach am Main

Müller-Westermeier G (2004) Statistical analysis of results of homogeneity testing and homogenization of long climatological time series in Germany. Fourth seminar for homogenization and quality control in climatological databases, Budapest, Hungary, 06-10 October 2003. World Climate Data and Monitoring Programme Series (WCDMP-No 56). WMO Technical Document, Bd. 1236. World Meteorological Organization, Geneva, Switzerland

Oberrheinische Universitäten (Basel, Freiburg, Straßburg, Karlsruhe) (Hrsg) (1996) REKLIP-Klimaatlas Oberrhein Mitte-Süd. vdf-Hochschulverlag, Zürich

Posselt R, Mueller RW, Stöckli R, Trentmann J (2012) Remote sensing of solar surface radiation for climate monitoring - the CM-SAF retrieval in international comparison. Remote Sensing of Environment 118:186-198. doi:10.1016/j.rse.2011.11.016

Rapp J (2000) Konzeption, Problematik und Ergebnisse klimatologischer Trendanalysen für Europa und Deutschland. Berichte des Deutschen Wetterdienstes, Bd. 212. Selbstverlag des Deutschen Wetterdienstes, Offenbach am Main

Rauthe M, Steiner H, Riediger U, Mazurkiewicz A, Gratzki A (2013) A Central European precipitation climatology. Part I: Generation and validation of a high resolution gridded daily data set (HYRAS). Meteorologische Zeitschrift 22(3):235-256

Scherrer SC, Appenzeller C, Liniger MA (2006) Temperature trends in Switzerland and Europe: implications for climate normal. International Journal of Climatology 26(5):565-580. doi:10.1002/joc.1270

Schneider-Carius K (1955) Wetterkunde Wetterforschung: Geschichte ihrer Probleme und Erkenntnisse in Dokumenten aus drei Jahrtausenden. Verlag Karl Alber, Freiburg/München

Schönwiese C-D, Janoschitz R (2008) Klima-Trendatlas Deutschland 1901-2000, 2. Aufl. Berichte des Instituts für Atmosphäre und Umwelt der Universität Frankfurt/Main, Bd. 4.

Schönwiese C-D, Staeger T, Trömel S (2004) The hot summer 2003 in Germany. Some preliminary results of a statistical time series analysis. Meteorol Z N F 13:323-327

Walter A, Keuler K, Jacob D, Knoche R, Block A, Kotlarski S, Müller-Westermeier $G$, Rechid D, Ahrens W (2006) A high resolution reference data set of German wind velocity 1951-2001 and comparison with regional climate model results. Meteorologische Zeitschrift 15(6):585-596

Wege K (2002) Die Entwicklung der meteorologischen Dienste in Deutschland. Geschichte der Meteorologie in Deutschland, Bd. 5. Selbstverlag des Deutschen Wetterdienstes, Offenbach am Main

Wijngaard JB, Klein Tank AMG, Konnen GP (2003) Homogeneity of 20th century European daily temperature and precipitation series. Int J Climatol 23:679-692

Winkler P (2006) Hohenpeißenberg 1781-2006: das älteste Bergobservatorium der Welt. Geschichte der Meteorologie in Deutschland 7:174

WMO (1959) Technical regulations. Volume 1: General meteorological standards and recommended practices. WMO Technical Document, Bd. 49. World Meteorological Organization, Geneva, Switzerland

Zolina O, Simmer C, Kapala A, Shabanov P, Becker P, Mächel H, Gulev S, Groisma $P$ (2014) New view on precipitation variability and extremes in Central Europe from a German high resolution daily precipitation dataset: Results from the STAMMEX project. Bulletin of the American Meteorological Society 95:995-1002. doi:10.1175/BAMS-D-12-00134.1 
Open Access Dieses Kapitel wird unter der Creative Commons Namensnennung 4.0 International Lizenz (http://creativecommons.org/ licenses/by/4.0/deed.de) veröffentlicht, welche die Nutzung, Vervielfältigung, Bearbeitung, Verbreitung und Wiedergabe in jeglichem Medium und Format erlaubt, sofern Sie den/die ursprünglichen Autor(en) und die Quelle ordnungsgemäß nennen, einen Link zur Creative Commons Lizenz beifügen und angeben, ob Änderungen vorgenommen wurden.

Etwaige Abbildungen oder sonstiges Drittmaterial unterliegen ebenfalls der genannten Creative Commons Lizenz, sofern sich aus der Abbildungslegende oder der Quellreferenz nichts anderes ergibt. Sofern solches Drittmaterial nicht unter der genannten Creative Commons Lizenz steht, ist eine Vervielfältigung, Bearbeitung oder öffentliche Wiedergabe nur mit vorheriger Zustimmung des betreffenden Rechteinhabers oder auf der Grundlage einschlägiger gesetzlicher Erlaubnisvorschriften zulässig. 